\title{
Roadmap for Computer-Aided Modeling of Theranostics and Related Nanosystems
}

\author{
Jozef Ulicny ${ }^{1}$ and Tibor Kozar $2,3,4, \star$ \\ ${ }^{1}$ Department of Biophysics, Institute of Physics, Safarik University, Kosice, Slovakia \\ ${ }^{2}$ Center for Interdisciplinary Biosciences, TIP-UPJS, Safarik University, Kosice, Slovakia \\ ${ }^{3}$ Institute of Experimental Physics, Slovak Academy of Sciences, Kosice, Slovakia \\ ${ }^{4}$ Laboratory of Information Technologies JINR, 141980 Dubna, Moscow Region, Russia
}

\begin{abstract}
Detailed understanding of the interactions of novel metal-containing nanoparticles with biological membranes, macromolecules and other molecular targets of the living cell is crucial for the elucidation of the biological actions of such functionalized nanosystems. We present here the construction and modeling of thiolate-protected gold clusters and the prediction of their static and dynamic properties.
\end{abstract}

\section{Introduction}

Nanotechnologies have significant influence on the recent research and development in many application areas. The biomedicine belongs to the most influenced fields. Thanks to the introduction of a new kind of nanoparticles (NPs) entitled theranostics (NP systems designed for combined diagnostics and therapeutic use), a new horizon was opened in imaging and treatment. Theranostic systems can be constructed from metallic core NPs functionalized with monolayer-covered bio-active molecules. Despite the recent "state of the art" level of molecular modeling methodologies and the number of articles published annually on noble-metal nanostructures, this particular field is still in the discovery phase [1]. In addition, the detailed understanding of the interactions of functionalized NPs with biological membranes, macromolecules and other entities of the living cells has crucial importance for the elucidation of the mechanisms governing the biological actions of such nanosystems. Insights into molecular details as to how these nanomaterials interact with subcellular nano-machinery of the cells can facilitate the design and engineering of a new generation of NPs [2]. We were interested in building and modeling thiolate-protected gold clusters and gold clusters functionalized with other biomolecules. We have computed their static and dynamic properties. Due to the system size and the inherent difficulties of the metallic NP modeling, such a task is a formidable challenge. As a part of our modeling roadmap, we aim to build models for NP - chromatin fragments, resembling interactions of such particles with higher-order DNA structures of living cells. Reliable computer modeling of intra- and intermolecular interactions in NP-chromatin models is a very complex task due to the size of such systems. Accordingly, we proceeded in several modeling steps which are described below.

\footnotetext{
^e-mail: tibor@saske.sk;tibor.kozar@upjs.sk
} 




Figure 1. Schematic roadmap starting from structure generation. Due to the number of atoms and the complexity of the systems, the permanent data analysis and visualization are of key importance to the derivation of solutions

\section{Computational methodology}

Several different computational protocols were needed to build, compute, and analyse the nanosystems under study. Figure 1 represents the roadmap - interconnections between different tasks starting from structure generation (upper left box). Understanding the structural complexity of the studied systems (starting from gold-containing NPs to model theranostics up to building DNA-related biomacromolecular systems) required very different approaches (all-atom (AA) versus coarse-grained (CG) modeling). Accordingly, different programs (running on Windows, Linux, MacOS) and web services were used in order to fill the boxes shown in Figure 1. Table 1 summarizes the most important programs used in the presented study. Taking into account our former experience with GPGU computing [3], we preferred the GPGU versions of the listed programs where available.

\section{Results and discussion}

We were interested in functionalization of differently sized gold-core NPs (from 25 to more than 100 $\mathrm{Au}$ atoms). Based on the experimental availability of Au-related structural data the data of Murray et al [4] were used for the construction of Au-25 clusters and the data of Kornberg et al [5] for the Au102 clusters. The results of geometry optimization (carried out by the Forcite program of Materials Studio, see Table 1) of two differently functionalized $\mathrm{Au}_{25} \mathrm{~S}_{18}$ clusters are presented in Figure 2.

MD simulations (Forcite program of Materials Studio) of these nanoclusters confirmed the conformational stability of the systems. In addition to molecules shown in Fig. 2, we built and calculated several other NPs in two ways: by enlarging the number of core Au atoms and, changing the functionalizing coverage as well. Even more, we have included doxorubicin into the NP [6] as a known way of facilitating drug delivery.

In the next step, we were interested in the development of advanced models for biomacromolecular systems where we predict possible bindings of the generated NPs. For detailed atomistic modeling of functionalized gold NP-nucleosome interactions, we downloaded the structure of nucleosome core particle (NCP) resolved at $2.5 \AA$ resolution [7]. In accordance with our roadmap-scheme (Fig. 1), we ended up with minimized structure of NCP to which the gold-core NPs were docked. Figure 3 represents the lowest-energy structure obtained in this way. 
Table 1. Summary of computational tools used in presented study

\begin{tabular}{|l|l|}
\hline $\begin{array}{l}\text { PROGRAM or } \\
\text { WEB SERVICE: }\end{array}$ & Reference or provider: \\
\hline $\begin{array}{l}\text { Discovery Studio (a) } \\
\text { Materials Studio (b) }\end{array}$ & $\begin{array}{l}\text { (a) BIOVIA, Discovery Studio Modeling Environment (2015) } \\
\text { (b) Accelrys/BIOVIA Materials Studio v7.0, 2013 Dassault Systèmes, } \\
\text { San Diego, USA }\end{array}$ \\
\hline HEX & $\begin{array}{l}\text { D.W. Ritchie, Evaluation of protein docking predictions using Hex 3.1 in } \\
\text { CAPRI rounds 1 and 2, Proteins 52 (1) 98-106 (2003) }\end{array}$ \\
\hline ICM & $\begin{array}{l}\text { R.C. Stolz and T.C. Bishop, ICM Web: the interactive chromatin modeling } \\
\text { web server, Nucleic Acids Res. 38 (Web Server issue) W254-61 (2010) }\end{array}$ \\
\hline MAESTRO & $\begin{array}{l}\text { Maestro, version 10.1.012, in Schrödinger Release 2015-12015, } \\
\text { Schrödinger, LLC: New York, NY., USA }\end{array}$ \\
\hline NAMD (c) & $\begin{array}{l}\text { (c) L. Kale et al., NAMD2: Greater scalability for parallel molecular } \\
\text { dynamics, Journal of Computational Physics 151 (1) 283-312 (1999) } \\
\text { (d) W. Humphrey, A. Dalke, and K. Schulten, VMD: visual molecular } \\
\text { dynamics, Journal of Molecular Graphics 14 (1) 27-28, 33-38 (1996) }\end{array}$ \\
\hline PACKMOL & $\begin{array}{l}\text { L. Martinez et al., PACKMOL: a package for building initial configu- } \\
\text { rations for molecular dynamics simulations, Journal of Computational } \\
\text { Chemistry 30 (13) 2157-2164 (2009) }\end{array}$ \\
\hline VDNA & $\begin{array}{l}\text { T.C. Bishop, VDNA: the virtual DNA plug-in for VMD, Bioinformatics 25 } \\
\text { (23) 3187-3188 (2009) }\end{array}$ \\
\hline
\end{tabular}

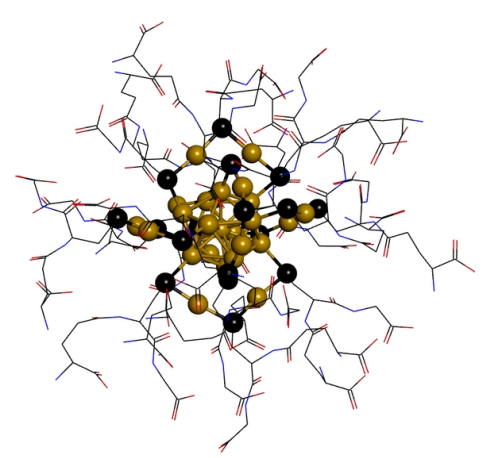

(A)

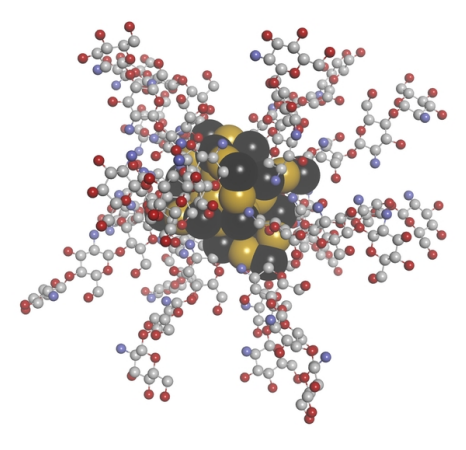

(B)

Figure 2. Glutathion and chitotriose functionalized NPs: (A) $\mathrm{Au}_{25} \mathrm{Glut}_{18}$ with the Au-S core shown in ball\&stick and glutathions in line representation; (B) $\mathrm{Au}_{25} \mathrm{Chito}_{18}$ with the $\mathrm{Au}-\mathrm{S}$ shown as $\mathrm{CPK}$ models and S-linked chitotriose molecules shown in ball\&stick representation. The $\mathrm{S}$ atoms are colored black.

The computer-aided modeling of chromosome fragments is a complex task due the size and related number of atoms of these systems. There are several computational resources helping the task, and we ended up with plenty of interesting coarse-grained structures. One of these structures is shown in Figure 3. Part of the molecule was updated by atomistic representation of histone in order to have better option to realistically dock the functionalized NP and get deeper insight into atomistic details of the intermolecular interactions. Due to the complexity/size of the system this modeling and the corresponding computations are not finalized yet. 


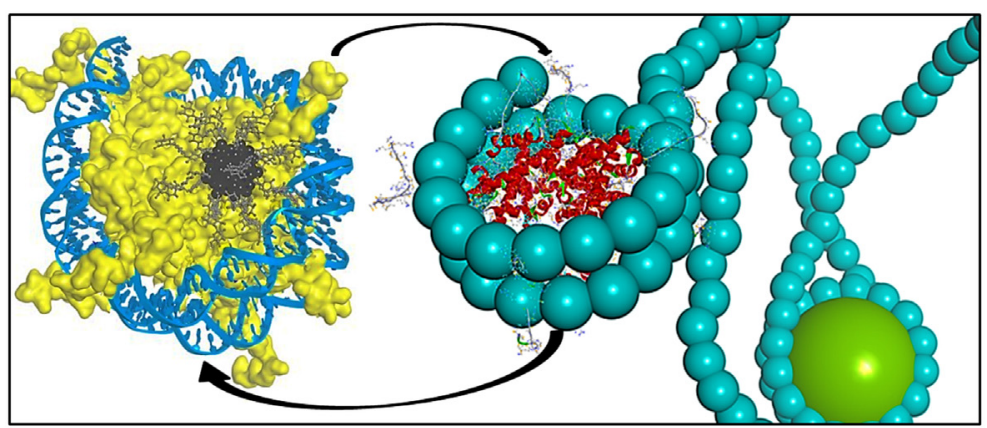

Figure 3. NCP in interaction with chitotriose functionalized gold NP (left; the NP is shown in black) and coarse-grained chromatin model with embedded all-atom histone (right). The arrows indicate the corresponding structural parts of $\mathrm{NCP} /$ chromatin. In coarse-grained model the medium size circles correspond to nucleotides. The largest circle corresponds to the second histone. Real molecular surface is shown for histone proteins (left).

\section{Conclusion}

Based on hardware and software availability, together with the gained experience in the generation of complex supramolecular assemblies, we were able to construct functionalized gold-NPs and calculate their static and dynamic properties. We proceeded to the modeling of their interactions with more complex macromolecular entities present in living cells like nucleosome particles. Such approaches will be of key importance for computer-aided modeling of biomarkers, as well as the interpretation of data for upcoming use in free-electron X-ray experiments.

\section{Acknowledgements}

This work was funded by the EU 7FP grant CELIM 316310. Supports from EU projects 2622012033 , 26210120002, 26110230097, and 26210120012 are gratefully acknowledged. We also would like to acknowledge Dr. Eva Valusova and Prof. Marian Antalik (IEP SAS) for experimental support and discussions on the functionalized NPs.

\section{References}

[1] S. Malola and H. Häkkinen, Europhysics News 46, 23 (2015)

[2] N. Yanamala, V. E. Kagan, and A. A. Shvedova, Adv. Drug. Deliv. Rev. 65, 2070 (2013)

[3] T. Kozar, in Lecture Notes in Computer Science: Mathematical Modeling and Computational Sciences 7125, (Springer Verlag, Heidelberg, Dordrecht, London, New York 2012) pp. 276

[4] M. W. Heaven, A. Dass, P. S. White, K. M. Holt, and R. W. Murray, J. Am. Chem. Soc. 130, 3754 (2008)

[5] P. D. Jadzinsky, G. Calero, C. J. Ackerson, D. A. Bushnell, and R. D. Kornberg, Science 318, 430 (2007)

[6] F. Wang, Y. C. Wang, S. Dou, M. H. Xiong, T. M. Sun, and J. Wang, ACS Nano 5, 3679 (2011)

[7] J. M. Harp, B. L. Hanson, D. E. Timm, and G. J. Bunick, Acta Crystallographica Section D, Biological Crystallography 56, 1513 (2000) 